\title{
Estudio de la Variación de Parámetros de Color del Yacón utilizando un Prototipo de Medición de Color y Temperatura
}

\author{
Alba Mery Garzón-García, Saúl Dussán-Sarria, Raúl Emilio Melo-Sevilla \\ Facultad de Ingeniería, Dpto. Ingeniería y Administración, Univ. Nacional de Colombia. Sede Palmira. A.A. \\ 237. Palmira, Valle del Cauca-Colombia. (e-mail: almgarzonga@unal.edu.co; sdussan@unal.edu.co; \\ remelos@unal.edu.co)
}

Recibido Feb. 15, 2018; Aceptado Abr. 17, 2018; Versión final Jun. 22, 2018, Publicado Dic. 2018

\begin{abstract}
Resumen
El objetivo de este trabajo fue proponer un prototipo de medición de color y temperatura construido con los sensores DHT11 de temperatura y TCS230-3200 de color conectados a un Arduino Nano, con una conexión serial se transfirió la información a un ordenador con los programas PXL-DAQ y Microsoft Exce ${ }^{\circledR}{ }^{2} 2013$, siendo las mediciones de color registradas en el modelo de color RGB para su conversión al sistema CIE L*a*b*. Un equipo de medición simultánea de color y temperatura a nivel piloto, como el que se propone, se convierte en una alternativa económica frente a los costosos equipos comerciales. Con el prototipo se analizó la pérdida de luminosidad y la variación temperatura del yacón (Smallanthus sonchifolius), y los cambios de índice de pardeamiento y diferencia de color. Estos mostraron un comportamiento similar al encontrado en la literatura.
\end{abstract}

Palabras clave: Smallanthus sonchifolius; prototipo de medición; luminosidad; índice de pardeamiento; diferencia de color.

\section{Evaluation of Yacon Luminosity with Color and Temperature Measurement Prototype}

\begin{abstract}
The objective of this work was to propose a color and temperature measurement prototype constructed with the temperature sensor DHT11 and the color sensor TCS230-3200 connected to an Arduino Nano, with a serial connection information was transferred to a computer with PXL-DAQ and Microsoft Excel $\AA^{2} 2013$ programs, the color measurements were recorded in RGB color model for a conversion to CIE $\mathrm{L}^{*} \mathrm{a}^{*} \mathrm{~b}^{*}$ system. A simultaneous color and temperature measurement equipment at pilot level, as the one proposed in this paper, becomes an economical alternative to costly commercial equipment. With the prototype the luminosity loss and changes of temperature of yacon (Smallanthus sonchifolius) were quantified, and the changes of browning indexes and color difference. These parameters presented a behavior similar to that found in the literature.
\end{abstract}

Keywords: Smallanthus sonchifolius; measurement prototype, luminosity, browning index, color difference. 


\section{INTRODUCCIÓN}

El color se define como la percepción humana de la luz reflejada por un objeto. Es un fenómeno que se genera cuando el cerebro procesa la luz captada a través de los ojos, y se ve afectado por variables como el objeto, el observador, el iluminante, la geometría óptica, el área, el fondo, la superficie, el brillo y la temperatura (Delmoro et al., 2010). La importancia del color en los alimentos radica en que es el primer atributo que valoran los consumidores en la elección de un producto. Asimismo, se relaciona con la determinación de la calidad e idoneidad de forma rápida, precisa y objetiva (Mathias-Rettig y Ah-Hen, 2014). La evaluación del color se realiza con sistemas visuales, en los cuales se analizan comparaciones con referencias de color bajo iluminación controlada o con colores estándar, implicando una inspección especializada y el entrenamiento de los observadores. También se mide con instrumentos como el colorímetro, el espectrofotómetro, el espectrocolorímetro y la visión computarizada (Pathare et al., 2013) como tecnología para adquirir y analizar una imagen con herramientas computacionales y electrónicas para obtener información o controlar procesos (Mery y Pedreschi, 2005). Por otro lado, la medición de temperatura en los alimentos es fundamental durante las operaciones agroindustriales. Para determinar este parámetro se utilizan los dispositivos de temperatura de fuerza (FTDs) divididos en bimetálicos y sistemas térmicos rellenos, y los dispositivos de temperatura eléctrica, como lo son los detectores de temperatura de resistencia (RTD), termopares, resistencias de silicio y semiconductores (Richardson, 2001).

A nivel de planta piloto o laboratorio es importante disponer de instrumentos y equipos que permitan medir diferentes atributos en los alimentos y que no demanden grandes costos. He ahí el porqué del desarrollo y diseño de prototipos a escala piloto que permitan automatizar y controlar procesos (Narendra y Hareesha, 2010). En este caso, de productos sensibles como la raíz reservante del yacón (Smallanthus sonchifolius), un vegetal que durante su pelado y procesamiento sufre de una rápida reacción enzimática al contacto de la pulpa con el oxígeno, debido a la actividad de la polifenoloxidasa y a su contenido de compuestos fenólicos (Rodrigues et al., 2014). Con la evaluación del color del yacón a diferentes temperaturas y empleando modelos cinéticos, es posible describir la degradación térmica del color a partir de la pérdida de luminosidad (Zhu et al., 2016). Los modelos cinéticos de degradación de componentes alimenticios son esenciales para el diseño de nuevos procesos y dan como resultado productos de alta calidad. Los parámetros cinéticos, entre ellos la constante de velocidad de reacción y la energía de activación, contribuyen en la predicción de la calidad final del producto y a minimizar los cambios no deseados en productos procesados (Urrea et al., 2012).

El objetivo de este trabajo fue utilizar un prototipo de medición de color y temperatura construido con sensores, tarjetas electrónicas de bajo costo y un sistema de adquisición de datos, para determinar la cinética de variación de la luminosidad, cambios de los valores del índice del pardeamiento y las diferencias de color en las raíces del yacón a las temperaturas de $10^{\circ} \mathrm{C}, 20^{\circ} \mathrm{C}$ y $30^{\circ} \mathrm{C}$.

\section{MATERIALES Y MÉTODOS}

Se detalla el funcionamiento del equipo empleado para la medición de color y temperatura, la adecuación del material vegetal, la evaluación de la cinética de variación de color del yacón por efecto de las variables ya mencionadas, así como el cálculo de los parámetros de color BI y $\Delta \mathrm{E}$. De igual forma, se describe cómo se llevó a cabo el análisis estadístico y se corroboró el ajuste del modelo cinético.

\section{Funcionamiento del prototipo de medición de color y temperatura}

Se observó inicialmente que los equipos que miden más de dos atributos son difíciles de conseguir, adaptar o no existen para ciertos proceso agroindustriales. En cuanto a la ciencia del color en los alimentos, se tiene que normalmente la medición de este parámetro se lleva a cabo con un colorímetro, el cual posee la desventaja de evaluar superficies menores a los $2 \mathrm{~cm}^{2}$, obteniendo medidas poco representativas y un complejo análisis global del alimento (León et al., 2006). Para mitigar el inconveniente anteriormente mencionado, investigadores crearon sistemas de visión computarizada incluyendo ordenadores, cámaras digitales y videocámaras (Saldaña et al., 2014), algoritmos lineales, cuadráticos y neurales, softwares especializados para segmentar las imágenes como Photoshop, Matlab e Image J (Saldaña et al., 2013). Sin embargo, en la literatura no se encontraban reportes específicos en relación al uso de sensores de fácil adquisición para evaluar el color de los alimentos. Siendo así, se pensó en un prototipo con elementos de bajo costo, que se pudieran programar en un lenguaje sencillo $\left(\mathrm{C}_{++}\right)$e integrar con softwares libres encontrados en Internet.

El prototipo de medición de color y temperatura en alimentos fue elaborado y ensayado en el Laboratorio de Frutas y Hortalizas de la Universidad Nacional de Colombia, Sede Palmira. El equipo constó de una caja con dimensiones $20 \mathrm{~cm} \times 20 \mathrm{~cm} \times 20 \mathrm{~cm}$ y paredes de color negro. Se adaptaron el sensor de temperatura DHT11 en la base y el sensor de color TCS230-3200 sobre el material biológico a evaluar, y fueron conectados a un 
Arduino Nano, el cual tenía el rol de tarjeta de adquisición de datos (DAQ). Con una conexión serial, se estableció la transferencia de los datos para ser procesados en un ordenador con la ayuda del programa PXLDAQ. Los valores obtenidos por medio del sistema de adquisición de datos, se llevaron a un archivo en Microsoft Exce ${ }^{\circledR} 2013$ para realizar la conversión del modelo de color RGB al sistema CIE L*a*b*.

\section{Conversión del sistema RGB a CIE L*a*b*}

El sensor TCS230-3200 detecta las medidas de color en el sistema RGB y estas son convertidas al sistema CIE L*a*b*. Según Saldaña et al. (2014), el primer paso de esta conversión, es transformar los valores RGB ( $R=$ rojo, $G=$ verde, $B=a z u l)$ en valores rgb como se nota en las Ecuaciones 1, 2 y 3, obteniendo la primera variación.

$$
\begin{aligned}
& \operatorname{VarR}=\mathrm{R} / 255 \\
& \operatorname{VarG}=\mathrm{G} / 255 \\
& \operatorname{VarB}=\mathrm{B} / 255
\end{aligned}
$$

En seguida fueron consideradas las condiciones a) y b) y aplicadas las ecuaciones 4 a la 9 .

a) Si VarR; VarG; VarB $\leq 0,404045$ entonces,

$$
\begin{aligned}
& \mathrm{r}=\operatorname{VarR} / 12,92 \\
& \mathrm{~g}=\operatorname{VarG} / 12,92 \\
& \mathrm{~b}=\operatorname{VarB} / 12,92
\end{aligned}
$$

b) Si VarR; VarG; VarB $>0,404045$ entonces,

$$
\begin{aligned}
& r=\left(\frac{\operatorname{VarR}+0,055}{1,055}\right)^{2,4} \\
& \mathrm{~g}=\left(\frac{\operatorname{VarG}+0,055}{1,055}\right)^{2,4} \\
& \mathrm{~b}=\left(\frac{\operatorname{VarB}+0,055}{1,055}\right)^{2,4}
\end{aligned}
$$

Posteriormente fue realizada la conversión de los parámetros rgb al sistema CIE XYZ, usando los coeficientes de la Ecuación 10, para un observador de $2^{\circ}$ y un iluminante D65.

$$
\left[\begin{array}{l}
\mathrm{X} \\
\mathrm{Y} \\
\mathrm{Z}
\end{array}\right]=100 \times\left[\begin{array}{lll}
0,4124 & 0,3576 & 0,1805 \\
0,2126 & 0,7152 & 0,0722 \\
0,0193 & 0,1192 & 0,9505
\end{array}\right]\left[\begin{array}{l}
\mathrm{r} \\
\mathrm{g} \\
\mathrm{b}
\end{array}\right]
$$

Luego fue realizada la conversión del sistema CIE XYZ al sistema CIE L*a*b* con una variación de los valores de $\mathrm{X}, \mathrm{Y}$ y $Z$; en donde los valores de $X_{n}, Y_{n}, Z_{n}$ para un observador de $2^{\circ}$ e iluminante $\mathrm{D} 65$ fueron respectivamente 95,$03 ; 100$ y 108,88 . Para esto se tuvieron las condiciones a) y b) y se aplicaron las ecuaciones 11 a la 16 .

a) $\mathrm{Si} \frac{\mathrm{X}}{\mathrm{X}_{\mathrm{n}}} ; \frac{\mathrm{Y}}{\mathrm{Y}_{\mathrm{n}}} ; \frac{\mathrm{Z}}{\mathrm{Z}_{\mathrm{n}}}>0,008856$ entonces;

$$
\begin{aligned}
& \operatorname{VarX}=\left(\frac{X}{X_{n}}\right)^{1 / 3} \\
& \operatorname{VarY}=\left(\frac{Y}{Y_{n}}\right)^{1 / 3} \\
& \operatorname{VarZ}=\left(\frac{Z}{Z_{n}}\right)^{1 / 3}
\end{aligned}
$$


b) $\mathrm{Si} \frac{\mathrm{X}}{\mathrm{X}_{\mathrm{n}}} ; \frac{\mathrm{Y}}{\mathrm{Y}_{\mathrm{n}}} ; \frac{\mathrm{Z}}{\mathrm{Z}_{\mathrm{n}}} \leq 0,008856$ entonces;

$$
\begin{aligned}
& \operatorname{VarX}=\left(7,787 \times \frac{X}{X_{n}}\right)+\frac{16}{116} \\
& \operatorname{Var} Y=\left(7,787 \times \frac{Y}{Y_{n}}\right)+\frac{16}{116} \\
& \operatorname{VarZ}=\left(7,787 \times \frac{Z}{Z_{n}}\right)+\frac{16}{116}
\end{aligned}
$$

Finalmente, se hallaron los valores del sistema CIE L*a*b* con las ecuaciones 17,18 y 19 .

$$
\begin{aligned}
& \mathrm{L} *=(116 \times \operatorname{VarY})-16 \\
& \mathrm{a} *=500 \times(\operatorname{VarX}-\operatorname{Var} Y) \\
& \mathrm{b} *=200 \times(\operatorname{Var} \mathrm{Y}-\operatorname{Var} \mathrm{Y})
\end{aligned}
$$

Evaluación de la cinética de variación de color en yacón

Fue utilizado $1 \mathrm{~kg}$ de la raíz yacón (Smallanthus sonchifolius) comprada en el mercado local. Se llevó a cabo la selección del material biológico con el fin de eliminar producto con evidentes daños mecánicos y una clasificación peso y tamaño, siendo este en media de $450 \mathrm{~g}$ por cada raíz y un diámetro aproximado de $8 \mathrm{~cm}$. Siguiendo la metodología de Saldaña et al. (2014), las raíces fueron lavadas con agua potable y detergente neutro para eliminar los rastros de suciedad. Luego, fueron inmersas en una solución a $100 \mathrm{ul} \mathrm{I}^{-1}$ de hipoclorito de sodio durante tres minutos antes de ser almacenado durante 18 horas a $4{ }^{\circ} \mathrm{C}$. Posteriormente y en el mismo instante de realizar la medición de las coordenadas de color, al material biológico le fue retirada la cáscara de forma manual y cortado en rodajas de $0,5 \mathrm{~cm}$ de diámetro, esta operación debe de realizarse rápidamente debido a que el oscurecimiento del yacón es acelerado.

\section{Calibración del prototipo de medición de color y temperatura}

Previamente a la evaluación del yacón, la temperatura se verificó con un termopar y la calibración del color del prototipo se realizó con un convertidor de color, una carta de códigos de color con valores de RGB en línea y las cartas de color PANTONE.

\section{Medición de color y temperatura del yacón}

La temperatura en el yacón se controló con el sensor de temperatura del prototipo y se dio inicio a la medición de color cuando la temperatura del producto alcanzó los $10^{\circ} \mathrm{C}$, registrando datos a cada 5 segundos de acuerdo a la programación de la tarjeta de adquisición de datos hasta que la superficie del yacón llegara a los $30^{\circ} \mathrm{C}$. El aumento gradual de la temperatura del fruto se logró por la ganancia de calor del mismo medio ambiente sin contar con ningún tipo de calentamiento ni control de esta variación de temperatura. El programa PLX-DAQ permitió que los datos se transfirieran a Microsoft Excel® 2013 para realizar la conversión del sistema RGB al sistema CIE $L^{*} a^{*} b$, el estudio de la cinética de variación de la luminosidad y el análisis estadístico.

Índice de pardeamiento $(B I)$

El índice de pardeamiento enzimático o browning index (BI) fue cuantificado a través del índice bioquímico (Lunadei et al., 2011) y Dussán-Sarria et al. (2017) utilizando las ecuaciones 20 y 21.

$$
\begin{aligned}
& \mathrm{BI}=\frac{(\mathrm{x}-0,31)}{0,17} \times 100 \\
& \mathrm{x}=\frac{\left(\mathrm{a}^{*}+1,75 \mathrm{~L}^{*}\right)}{\left(5,645 \mathrm{~L}^{*}+\mathrm{a}^{*}-3,012 \mathrm{~b}^{*}\right)}
\end{aligned}
$$

\section{Diferencia de color}

La diferencia de color $(\Delta E)$ fue calculada entre los valores $L^{*} a^{*} b^{*}$ obtenidos con el prototipo y su comparación con los valores estándares en el sistema CIE $L^{*} a^{*} b^{*}$. Si $\Delta L^{*}$ es positivo, la muestra es más luminosa que la 
estándar. Si $\Delta \mathrm{a}^{*}$ es negativo, sería más verde o menos rojo. Si $\Delta \mathrm{b}^{*}$ es positivo, la muestra es más amarilla o menos azul que la estándar. El valor de $\Delta \mathrm{E}$ fue determinado con las ecuaciones $22,23,24$ y 25 , en donde el subíndice 1 es para la muestra estándar y el 2 es para las rodajas de yacón evaluadas (Jha, 2010).

$$
\begin{aligned}
& \Delta \mathrm{L}^{*}=\mathrm{L}_{2}^{*}-\mathrm{L}_{1}^{*} \\
& \Delta \mathrm{a}^{*}=\mathrm{a}_{2}^{*}-\mathrm{a}_{1}^{*} \\
& \Delta \mathrm{b}^{*}=\mathrm{b}_{2}^{*}-\mathrm{b}_{1}^{*} \\
& \Delta \mathrm{E}=\sqrt{\left(\mathrm{L}_{2}^{*}-\mathrm{L}_{1}^{*}\right)^{2}+\left(\mathrm{a}_{2}^{*}-\mathrm{a}_{1}^{*}\right)^{2}+\left(\mathrm{b}_{2}^{*}-\mathrm{b}_{1}^{*}\right)^{2}}
\end{aligned}
$$

\section{Cálculo de la cinética de variación de luminosidad}

Para las temperaturas de análisis $10^{\circ} \mathrm{C}, 20^{\circ} \mathrm{C}$ y $30^{\circ} \mathrm{C}$ fueron calculadas las constantes de variación y energía de activación con la luminosidad $\left(L^{*}\right)$. Según Ordoñez-Santos et al. (2014), para determinar el cambio de color de los alimentos como función del tratamiento térmico, muchas ecuaciones han sido publicadas en la literatura, donde la variación del factor $\mathrm{C}$ en el tiempo puede ser representado de acuerdo a la ecuación 26.

$$
\frac{\mathrm{dC}}{\mathrm{dt}}=-\mathrm{kC} \mathrm{C}^{\mathrm{n}}
$$

En donde $\mathrm{k}$ se refiere a la constante cinética $\left(\mathrm{s}^{-1}\right)$, C es la medida de color a un tiempo t y $\mathrm{n}$ el número de orden de reacción. Para la mayoría de los alimentos, la dependencia con el tiempo está descrita por cinética de orden cero o de primer orden, y se puede cuantificar según la ecuación de Arrhenius (ecuación 27).

$$
\mathrm{k}=\mathrm{e}^{-\frac{\mathrm{Ea}}{\mathrm{RT}}}
$$

En donde Ea es la energía de activación (J), R la constante de los gases ideales $(8,31434 \mathrm{~J} / \mathrm{mol} \mathrm{k})$ y $\mathrm{T}$ es la temperatura absoluta $(\mathrm{k})$.

\section{Factor $Q_{10}$}

Según Carrillo et al. (2016), el parámetro adimensional Q10 se define como la razón entre la constante de velocidad de reacción de pardeamiento a una temperatura $(T)$ y la constante de velocidad de reacción de pardeamiento a una temperatura $T+10^{\circ} \mathrm{C}$. Se representa con la ecuación 28 , siendo $\mathrm{k}_{\mathrm{T}}$ la constante de velocidad de reacción a la temperatura $T$ y $\mathrm{k}_{\mathrm{T}+10}$ la constante de velocidad de reacción a la temperatura $\mathrm{T}+$ $10^{\circ} \mathrm{C}$.

$$
\mathrm{Q}_{10}=\frac{\mathrm{k}_{\mathrm{T}+10}}{\mathrm{k}_{\mathrm{T}}}
$$

\section{Análisis estadístico}

La evaluación de color del yacón fue realizada por triplicado a las temperaturas de análisis $10^{\circ} \mathrm{C}, 20^{\circ} \mathrm{C}$ y $30^{\circ} \mathrm{C}$. Asimismo, se llevó a cabo un ANOVA de un factor y con test de rango múltiple Duncan (IBM SPSS Statistics Subscription Beta Preview) para los datos de $L^{*}$, índice de pardeamiento y diferencia de color. Fue realizada una regresión lineal para hallar las velocidades de reacción y la energía de activación a partir de la variación de la luminosidad, y se estableció el ajuste del modelo cinético calculando el coeficiente de determinación, error medio absoluto (MAE) y error cuadrático medio (MSE).

\section{RESULTADOS Y DISCUSIÓN}

Se presentan los resultados y discusión en las subsecciones mencionadas seguidamente: cinética de variación de luminosidad en yacón, índice de pardeamiento y diferencia de color.

\section{Luminosidad e Índice de pardeamiento del yacón}

En la Tabla 1 se observan los valores medios de la coordenada $L^{*}$ o luminosidad de las rodajas de yacón siendo estadísticamente diferentes a $p<0,05$ en las tres temperaturas de $10^{\circ} \mathrm{C}, 20^{\circ} \mathrm{C}$ y $30^{\circ} \mathrm{C}$ con valores de $L^{*}$ de $79,4 \pm 1,44,74,54 \pm 1,10$ y $67,67 \pm 0,69$ respectivamente. La diminución de los valores de luminosidad a medida que se eleva la temperatura del yacón indica un efecto notorio causado por el incremento 
oscurecimiento enzimático a través del tiempo. De la misma forma existe una diferencia significativa $(\mathrm{p}<0,05)$ entre los valores de los índices de pardeamiento (BI) a las 3 temperaturas evaluadas con valores $\mathrm{BI}$ de $9,56 \pm 0,95,13,10 \pm 1,61$ y $17,02 \pm 0,87$ respectivamente. El aumento del grado de oscurecimiento evidenciado a su vez por la disminución de la luminosidad indica que hubo un cambio significativo en el color del yacón cortado a medida que se incrementa la temperatura en el material biológico (Tabla 1).

Tabla 1: Efecto de la temperatura en los valores de luminosidad $\left(\mathrm{L}^{*}\right)$ e índice de pardeamiento (BI) del yacón

\begin{tabular}{ccc}
\hline & \multicolumn{1}{c}{$10^{\circ} \mathrm{C}$} & $\mathrm{BI}$ \\
\hline Tiempo (s) & $\mathrm{L}^{*}$ & $8,94 \pm 1,51$ \\
\hline 0 & $80,78 \pm 2,45$ & $8,88 \pm 2,12$ \\
120 & $80,14 \pm 3,05$ & $9,52 \pm 2,55$ \\
180 & $79,21 \pm 3,48$ & $10,92 \pm 1,62$ \\
Promedio & $77,47 \pm 3,42$ & $9,56 \pm 0,95^{\mathrm{A}}$ \\
\hline & $79,40 \pm 1,44^{\mathrm{C}}$ & \\
\hline Tiempo (s) & $20^{\circ} \mathrm{C}$ & $\mathrm{BI}$ \\
\hline 2150 & $\mathrm{~L}^{*}$ & $11,88 \pm 2,05$ \\
2210 & $75,76 \pm 3,01$ & $12,16 \pm 1,07$ \\
2270 & $75,06 \pm 2,36$ & $12,95 \pm 1,84$ \\
2330 & $73,08 \pm 3,41$ & $15,41 \pm 3,30$ \\
Promedio & $74,24 \pm 1,69$ & $13,10 \pm 1,61^{\mathrm{B}}$ \\
\hline & $30{ }^{\circ} \mathrm{C}$ & \\
\hline Tiempo (s) & $\mathrm{L}^{*}$ & $\mathrm{Bl}$ \\
\hline 3520 & $68,56 \pm 5,00$ & $16,09 \pm 3,93$ \\
3580 & $67,84 \pm 5,46$ & $16,81 \pm 5,02$ \\
3640 & $67,34 \pm 5,84$ & $17,00 \pm 5,38$ \\
3700 & $69,95 \pm 4,28$ & $18,19 \pm 4,47$ \\
Promedio & $67,67 \pm 0,69^{\mathrm{a}}$ & $17,02 \pm 0,87^{\mathrm{C}}$ \\
\hline
\end{tabular}

Según Ding y Ling (2014), la medición del pardeamiento de un fruto es más efectivo con las ecuaciones derivadas del espacio de color que visualizando el área o superficie en donde se desarrolla este proceso. Por otro lado, Fante et al. (2012) argumentan que el BI puede ser correlacionado con la actividad de la enzima polifeniloxidasa (PPO) en la raíz reservante del yacón. A pesar de que la publicación de Ding y Ling (2014) hace referencia al banano y no al yacón, junto con la publicación de Fante et al. (2012), demuestran que si es posible obtener una cinética de degradación del color ocasionada por la PPO en yacón, sin embargo, en el presente trabajo no fue determinada la actividad de la PPO ni los coeficientes de correlación de esta enzima con los parámetros de color $\mathrm{L}^{*}, \mathrm{a}^{*}, \mathrm{~b}^{*}, \mathrm{BI}$, simplemente se estudió la cinética de variación de la luminosidad en yacón y los cambios de $\mathrm{BI}$ y color aducidos al efecto de la temperatura.

\section{Cinética de variación de luminosidad}

Fue considerada una cinética de primer orden debido al rápido cambio en el tiempo de los valores de luminosidad como se observa en la Figura 1, lo cual se corroboró con coeficientes de determinación mayores a 0,9 y valores bajos para el MAE y MSE (Tabla 2). Fueron determinadas las constantes cinéticas similares de $0,00023 \mathrm{~s}^{-1}, 0,00019 \mathrm{~s}^{-1}$ y $0,00013 \mathrm{~s}^{-1}$ para las temperaturas de $10^{\circ} \mathrm{C}, 20^{\circ} \mathrm{C}$ y $30^{\circ} \mathrm{C}$ respectivamente. La energía de activación calculada fue de $19,741 \mathrm{~J} \mathrm{~mol}^{-1}$, un valor elevado debido al cambio de color del yacón en corto tiempo y el efecto notorio de la temperatura en el oscurecimiento. En un estudio se encontró una energía de activación igual a $14,7 \mathrm{~kJ} / \mathrm{mol}$ al someter rodajas de yacón sometidas a un tratamiento térmico entre $60{ }^{\circ} \mathrm{C}$ y $80^{\circ} \mathrm{C}$ por 5 a 25 minutos, indicando que los parámetros de estabilidad del color son más sensibles a la temperatura (Zhu et al., 2016). El factor $Q_{10}$ se disminuyó a medida que se transcurrió el tiempo de evaluación y se aumentaba la temperatura. Entre las temperaturas $10^{\circ} \mathrm{C}$ y $20^{\circ} \mathrm{C}$ el $\mathrm{Q}_{10}$ fue de 0,84 ; mientras que entre $20^{\circ} \mathrm{C}$ y $30^{\circ} \mathrm{C}$ fue de 0,69 (Tabla 2). Es decir entre $20^{\circ} \mathrm{C}$ y $30^{\circ} \mathrm{C}$ el pardeamiento enzimático disminuyó el $17,7 \%$. Este parámetro es importante en un modelo para predecir la calidad de un alimento y aceleración del deterioro por cada $10^{\circ} \mathrm{C}$ de temperatura que se aumente (Oliveira et al., 2012). 
Tabla 2: Parámetros cinéticos de la variación de la luminosidad debida al efecto de la temperatura en el yacón

\begin{tabular}{cccccccc}
\hline Temperatura $\left({ }^{\circ} \mathrm{C}\right)$ & $\mathrm{k}\left(\mathrm{s}^{-1}\right)$ & $\mathrm{R}^{2}$ & $\mathrm{MAE}$ & $\mathrm{MSE}$ & $\mathrm{Q}_{10}$ & $\mathrm{Ea}\left(\mathrm{J} \mathrm{mol}^{-1}\right)$ & $\mathrm{R}^{2}$ \\
\hline 10 & 0,00023 & 0,95 & 0,0036 & $1,32 \mathrm{E}-05$ & - & & \\
20 & 0,00019 & 0,99 & 0,0007 & $6,31 \mathrm{E}-07$ & 0,84 & 19741 & 0,95 \\
30 & 0,00013 & 0,98 & 0,0012 & $1,49 \mathrm{E}-06$ & 0,69 & & \\
\hline
\end{tabular}

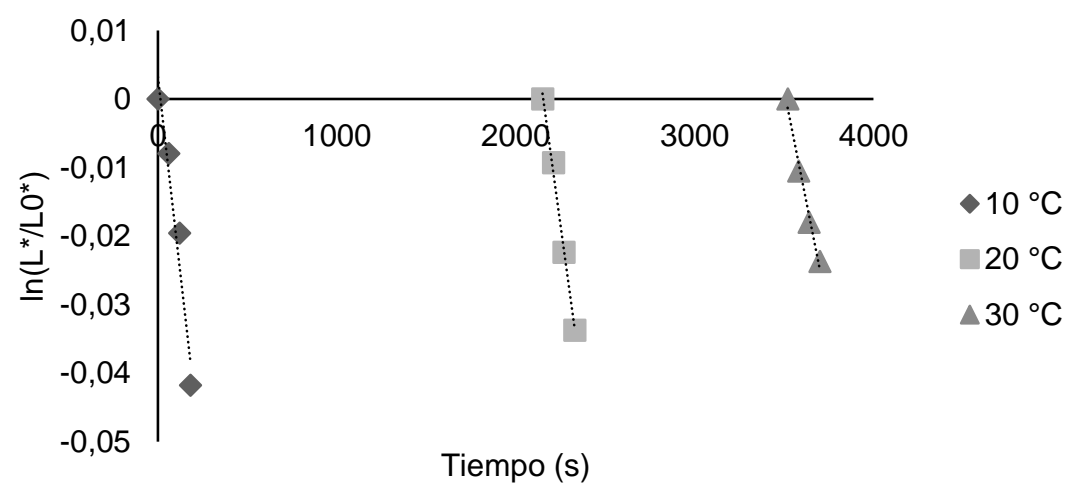

Fig. 1: Cinética de variación de primer orden en la luminosidad de rodajas de yacón a $10^{\circ} \mathrm{C}, 20^{\circ} \mathrm{C}$ y $30^{\circ} \mathrm{C}$

Diferencia de color

Los valores estándar para $L^{*}, a^{*}$ y $b^{*}$ fueron $80,78,6,03$ y 2,90, respectivamente, registrados con el prototipo al inicio de la medición de color del yacón. Se observó que la diferencia de color $(\Delta \mathrm{E})$ fue aumentando con el incremento de la temperatura del vegetal, encontrándose valores medios estadísticamente diferentes a $\mathrm{p}<0,05$, de $2,24 \pm 1,52,7,49 \pm 1,20$ y $14,89 \pm 0,80$ para $10^{\circ} \mathrm{C}, 20^{\circ} \mathrm{C}$ y $30^{\circ} \mathrm{C}$ respectivamente. Esta diferencia de color es conducente con la pérdida de $L^{*}$ e incremento de $B \mathrm{~B}$ ya discutido anteriormente. Las rodajas de yacón se tornaron entre rojizas y pardas (Tabla 3). Estos mismos resultados se encontraron en el estudio de Saldaña et al. (2014), quienes diseñaron, implementaron y calibraron un sistema de visión por computador (CVS) aplicado a rodajas de yacón mínimamente procesadas.

Tabla 3: Diferencia de color del yacón en rodajas

\begin{tabular}{|c|c|c|c|c|}
\hline \multicolumn{5}{|c|}{$10^{\circ} \mathrm{C}$} \\
\hline Tiempo (s) & $\Delta \mathrm{L}^{*}$ & $\Delta a^{*}$ & $\Delta \mathrm{b}^{*}$ & $\Delta \mathrm{E}$ \\
\hline 60 & $-0,64$ & 0,39 & $-0,40$ & 0,85 \\
\hline 120 & $-1,57$ & 1,12 & $-0,50$ & 1,99 \\
\hline 180 & $-3,31$ & 1,99 & $-0,17$ & 3,86 \\
\hline Promedio & $-1,84 \pm 1,35$ & $1,17 \pm 0,80$ & $-0,36 \pm 0,80$ & $2,24 \pm 1,52^{a}$ \\
\hline \multicolumn{5}{|c|}{$20^{\circ} \mathrm{C}$} \\
\hline Tiempo (s) & $\Delta \mathrm{L}^{*}$ & $\Delta a^{*}$ & $\Delta \mathrm{b}^{*}$ & $\Delta \mathrm{E}$ \\
\hline 2150 & $-5,02$ & 3,58 & $-0,72$ & 6,21 \\
\hline 2210 & $-5,72$ & 3,72 & $-0,67$ & 6,86 \\
\hline 2270 & $-6,70$ & 4,30 & $-0,62$ & 7,98 \\
\hline 2330 & $-7,54$ & 4,73 & 0,70 & 8,93 \\
\hline Promedio & $-6,24 \pm 1,10$ & $4,08 \pm 0,53$ & $-0,33 \pm 0,69$ & $7,49 \pm 1,20^{\mathrm{b}}$ \\
\hline \multicolumn{5}{|c|}{$30^{\circ} \mathrm{C}$} \\
\hline Tiempo (s) & $\Delta \mathrm{L}^{*}$ & $\Delta a^{*}$ & $\Delta \mathrm{b}^{*}$ & $\Delta \mathrm{E}$ \\
\hline 3520 & $-12,22$ & 6,70 & $-0,85$ & 13,96 \\
\hline 3580 & $-12,94$ & 6,81 & $-0,61$ & 14,63 \\
\hline 3640 & $-13,44$ & 6,83 & $-0,60$ & 15,09 \\
\hline 3700 & $-13,83$ & 7,76 & $-0,42$ & 15,86 \\
\hline Promedio & $-13,11 \pm 0,69$ & $7,02 \pm 0,49$ & $-0,62 \pm 0,18$ & $14,89 \pm 0,80^{c}$ \\
\hline
\end{tabular}




\section{CONCLUSIONES}

El prototipo creado para la medición de color y temperatura funcionó durante el estudio de cinética de variación de los valores de luminosidad, cambios en el índice de pardeamiento y cambios en la deferencia de color del yacón cortado. A través del prototipo fue posible evidenciar las variaciones de los parámetros de color $\mathrm{L}^{*}, \mathrm{BI}$ y $\Delta \mathrm{E}$ obteniendo resultados similares a varios autores, evidenciando así, la pérdida de luminosidad, el aumento del índice de pardeamiento y la notable diferencia de color en el yacón mínimamente procesado. El prototipo creado a nivel de laboratorio es un instrumento potencial de medida y control de calidad de los alimentos cuando el color y/o la temperatura tienen efecto directo sobre un alimento.

\section{REFERENCIAS}

Carrillo, G. L. E., M. D. P. Duque, Z. D. E. Martínez, D. Acevedo y P. M. Montero, Estudio de la vida útil de jamón de cerdo mediante pruebas aceleradas, ISSN: 0378-1844, Interciencia, 41(7), 488-491 (2016)

Delmoro, J., D. Muñoz, V. Nadal, A. Clementz y V. Pranzetti, El Color En Los Alimentos: Determinación De Color En Mieles, ISSN: 0329-3475, Invenio, 13(25), 145-152 (2010)

Ding, P. e Y. S. Ling, Browning assessment methods and polyphenol oxidase in UV-C irradiated Berangan banana fruit, ISSN: 2231-7546, Int. Food Res J., 21(4), 1667-1674 (2014)

Dussán-Sarria, S., A. F. Gaona-Acevedo y J. I. Hleap-Zapata, Efecto del Uso de Antioxidantes en Plátano Verde Dominico Hartón (Musa AAB Simmonds) Cortado en Rodajas, doi: 10.4067/S0718-07642017000400002, Inf. Tecnol., 28(4), 3-10 (2017)

Fante, L., C. F. Scher, C. P. Z. Noreña y A. O. Rios, Study of enzyme inactivation using steam in yacon (Smallanthus sonchifolius) roots, doi:10.1111/j.1745-4549.2011.00609.x, J. Food Process Preserv., 37(1), 16-24 (2013)

Jha, S. N., Colour Measurements and Modeling, in Nondestructive Evaluation of Food Quality by S. N. Jha, $1^{\text {a }}$ Ed., Springer-Verlag, 17-40, Berlín, Alemania (2010)

Lunadei, L., P. Galleguillos, B. Diezma, L. Lleó y L. Ruiz-Garcia, A multispectral vision system to evaluate enzymatic browning in fresh-cut apple slices, doi: 10.1016/j.postharvbio.2011.02.001, Postharvest Biol. Technol., 60(3), 225-234 (2011)

Mathias-Rettig, K. y K. Ah-Hen, El color en los alimentos un criterio de calidad medible, doi: 10.4206/agrosur.2014.v42n207, Agro Sur, 42(2), 39-48 (2014)

Mery, D. y F. Pedreschi, Segmentation of colour food images using a robust algorithm, doi: 10.1016/j.jfoodeng.2004.04.001, J. Food Eng., 66(3), 353-360 (2005)

Narendra, V. G. y K. S. Hareesha, Quality inspection and grading of agricultural and food products by computer vision- $A$ Review, doi: 10.5120/612-863, Int. J. Comput. Appl., 2(1), 43-65 (2010)

Oliveira, A.D.N., A. M. Ramos, V.P. R. Minim y J.B.P. Chaves, Sensory stability of whole mango juice: influence of temperature and storage time, doi: 10.1590/S0101-20612012005000115, Food Sci. Technol (Campinas), 32(4), 819-825 (2012)

Ordoñez-Santos, L.E., L.M. Tanganan y G.X. Mendez-Molano, A study of degradation kinetics regarding green peppers'(Capsicum spp.) surface colour, doi: 10.22579/20112629.276, Orinoquia, 18(1), 15-20 (2014)

Pathare, P. B., U. L. Opara y F. A. J. Al-Said, Colour measurement and analysis in fresh and processed foods: a review, doi: 10.1007/s11947-012-0867-9, Food Bioprocess Tech., 6(1), 36-60 (2013)

Richardson, P. (Ed.), Thermal technologies in food processing, 57-61, Taylor \& Francis, Padstow, Inglaterra (2001)

Rodrigues, O. R. L., E. R., Asquieri y D. C. Orsi, Prevention of enzymatic browning of yacon flour by the combined use of anti-browning agents and the study of its chemical composition, doi: 10.1590/fst.2014.0045, Food Sci. Technol (Campinas), 34(2), 275-280 (2014)

Saldaña, E., R. Siche, W. Castro, R. Huamán y R. Quevedo, Measurement parameter of color on yacon (Smallanthus sonchifolius) slices using a computer vision system, doi: 10.1016/j.Iwt.2014.06.037, LWT-Food Sci Technol, 59(2), 12201226 (2014)

Saldaña, E., R. Siche, M. Luján y R. Quevedo, Review: computer vision applied to the inspection, and quality control of fruits and vegetables, doi: 10.1590/S1981-67232013005000031, Braz. J. Food Technol., 16(4), 254-272 (2013)

Urrea, D., V. Eim, C. Roselló y S. Simal, Modelos cinéticos de degradación de carotenoides, polifenoles y actividad antioxidante durante el secado convectivo de zanahoria (Daucus carota V. Nantesa), ISSN 2027-291X, Alimentos Hoy, 21(27), 68-101 (2012)

Zhu, S., F. Hu, Q. Zhang, Y. Yu y H. S. Ramaswamy, A Novel Color Parametric Technique for Kinetic Evaluation of Thermal Degradation and Stability of Fresh-Cut Yacon Slices, doi: 10.1515/ijfe-2016-0144, INT J FOOD ENG, 12(8), $729-737$ (2016) 